\title{
Influence of Mechanical Treatment of the Z-Surface of Lithium Niobate on the Properties of X-ray Pyroelectric Source
}

\author{
O.O. Ivashchuk ${ }^{1}$, A.A. Klenin ${ }^{1}$, A.S. Kubankin ${ }^{1,2}$, A.N. Oleinik ${ }^{1,3}$, A.V. Shchagin ${ }^{1,4}$ \\ ${ }^{1}$ Belgorod State National Research University, 85, Pobeda Str., Belgorod, Russian Federation \\ ${ }^{2}$ Lebedev Physical Institute RAS, 53, Leninskiy Prosp., Moscow, Russian Federation \\ ${ }^{3}$ John Adams Institute at Royal Holloway, University of London, Egham, UK \\ ${ }^{4}$ Kharkiv Institute of Physics and Technology, Kharkiv, Ukraine
}

(Received 04 September 2018; revised manuscript received 07 December 2018; published online 18 December 2018)

\begin{abstract}
The effect of mechanical treatment of the $Z$-surface of a lithium niobate single crystal on the properties of a pyroelectric X-ray source was experimentally investigated. In the experiment, the properties of the source were measured with a roughness of the $Z$-surface of the crystal in the range $3-8 \mu \mathrm{m}$. As a result, the effect of the surface roughness of the pyroelectric crystal on the process of X-ray generation with a pyroelectric effect is shown.
\end{abstract}

Keywords: $Z$-surface, Pyroelectric crystal, Treatment, Mechanical properties.

\section{INTRODUCTION}

Single crystals of lithium niobate $\left(\mathrm{LiNbO}_{3}\right)$ are widely used in pyroelectric sources of X-ray radiation [1, 2]. The typical size of a pyroelectric sample of the cylindrical or cubic shape used in the sources is of the order of $1 \mathrm{~cm}$. Such a single crystal of sufficiently compact dimension is capable of generating a electric potential of about $50-100 \mathrm{kV}$ when the temperature of the crystal changes by $30-50{ }^{\circ} \mathrm{C}$ under vacuum condition (residual gas pressure 0.1-10 mTorr) due to the pyroelectric effect. Depending on the polarity of charge on the $Z$-surface of the crystal, electrons are emited from its surface and accelerated towards the grounded conductor located opposite (the so-called target) or accelerated towards the $Z$-surface from the surrounding space while target and opposite $Z$-surface of the crystal is grounded.

The only pyroelectric source currently available on an industrial scale, Amptek COOL-X (USA), allows generating radiation with an energy of up to $35 \mathrm{keV}$ and an intensity of up to $10^{7}$ quanta per second in peak [3]. However, such characteristics are far from the limit for pyroelectric sources. At present, the possibility of increasing the yield and energy of X-ray radiation generated by a pyroelectric source is being actively investigated [4]. One of such possibilities is the creation of a roughness on the $Z$-surface of the pyroelectric crystal, on which the potential is formed, by means of mechanical sanding of the surface. This kind of treatment can lead to the creation of a structure with microprotrusions that allow locally increasing the electric field, and as a consequence, enhancing the emission of electrons and their energy. Earlier, it was shown that sanding of working surface of the crystal using sandpaper with an average grain size of about $7 \mu \mathrm{m}$ reduces both the endpoint energy (and hence the value of generated electric potential) and the intensity of generated radiation [5]. However, an increase in the X-ray yield was observed in individual measurements nevertheless, but this phenomenon was not systematic.

This paper aims to investigate in more detail the possibility of improving the characteristics of a pyroe- lectric X-ray source by treating the free $Z$-surface of the crystal with sandpaper.

\section{OBJECTS AND METHODS}

The pyroelectric lithium niobate crystal $\left(\mathrm{LiNbO}_{3}\right)$ of cylindrical shape with a diameter of $20 \mathrm{~mm}$ and a thickness of $10 \mathrm{~mm}$ along the $\mathrm{z}$ axis was taken for the test. The $Z$-surface of crystal was attached to the duralumin radiator of the same size as crystal. In the radiator, a hole was drilled to accommodate a K-type thermocouple closer to the surface of the crystal. The assembly of radiator and crystal was fixed with an epoxy adhesive to the Peltier element and its radiator located in the cylindrical vacuum chamber. The distance between the crystal and the target was about $12 \mathrm{~mm}$. The scheme of the experiment is shown in Fig. 1.

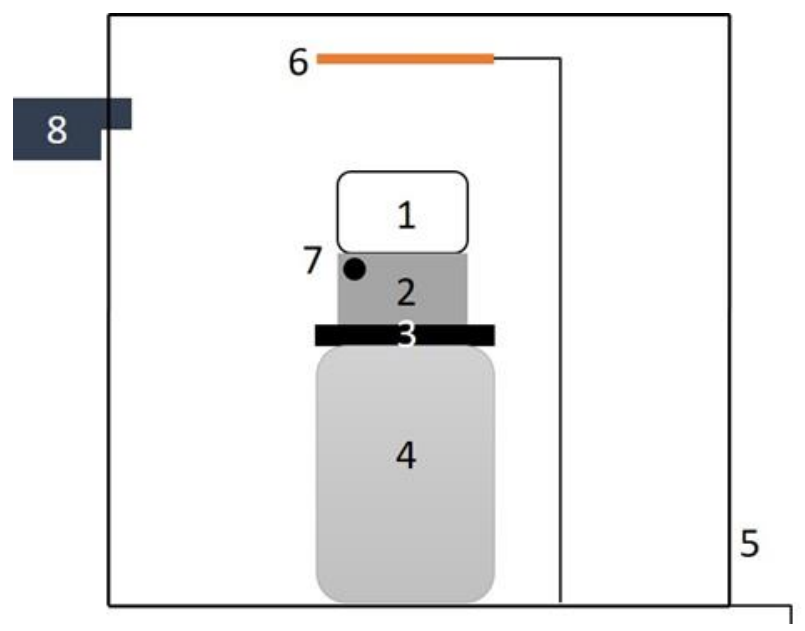

Fig. 1-The scheme of the experiment: 1 - pyroelectric crystal, 2 - radiator, 3 - Peltier element, 4 - radiator of Peltier element, 5 - wall of vacuum chamber, 6 - target, 7 - thermocouple, 8 - X-ray detector

The experiment consisted in measuring the X-ray spectrum during cyclic heating and cooling of the crys- 
tal at a residual gas pressure of 1 mTorr. Five thermal cycles of heating from $25^{\circ} \mathrm{C}$ to $60^{\circ} \mathrm{C}$ and cooling from $60{ }^{\circ} \mathrm{C}$ to $25^{\circ} \mathrm{C}$ without interruption after mechanical sanding with the sandpaper of a certain grain size were carried out each time.

The X-ray spectrum was measured using an X-ray semiconductor X-ray detector Amptek CdTe XR-100T. The detector was located on the flange of vacuum chamber at a level in the middle between the target and the free $Z$-surface of crystal, the distance from the entrance window of detector to the symmetry axis of the chamber (and the pyroelectric source) was about $350 \mathrm{~mm}$. Thus, with the help of the detector it became possible to detect X-ray radiation from both the target and the crystal.

First of all, images of the free $Z$-surface were obtained using of the Olympus GX-41 optical microscope and then the X-ray yield was measured during five thermal cycles. After the $Z$-surface was machined with the sandpaper with average grain size of $36 \mu \mathrm{m}$, then the surface relief was recorded with the optical microscope, and then the X-ray spectrum was measured for five thermal cycles. Studies were made in this mode after treating with sandpaper with a successively decreasing average grain size: $18 \mu \mathrm{m}, 9 \mu \mathrm{m}, 6 \mu \mathrm{m}, 3 \mu \mathrm{m}$. After carrying out these studies, the surface of crystal was treated with a polishing paste, recording of the surface relief and measuring of the X-ray yield was also carried out.

\section{RESULTS AND DISCUSSION}

Below, in Fig. 2, the results of the investigation of the state of crystal surface are presented - photographs obtained using an optical microscope. In the photo (a) and (b), one can see separate areas of protrusions. Also dimensions of the protrusions are reduced with a decrease in the average grain size. Besides, a lot of scratches are appeared after processing with sandpaper. After polishing paste processing (grain size less than $1 \mu \mathrm{m}$ ), the surface relief becomes more flat, most of the scratches and protrusions become invisible, as well as before machining. Thus, mechanical treatment significantly changes the surface relief.

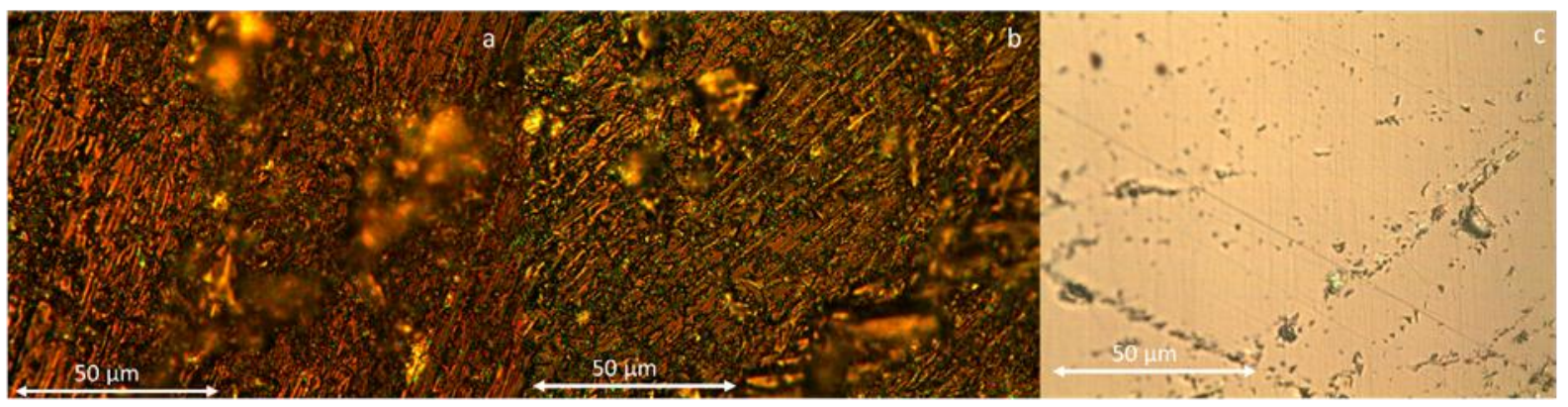

Fig. 2 - Photos of the free z-surface obtained using an optical microscope. (a) - after sandpaper treatment with an average grain size of $18 \mu \mathrm{m}$, (b) - after sandpaper treatment with an average grain size of $6 \mu \mathrm{m}$, (c) - after treatment with a polishing paste. Photos (a) and (b) are made in polarized light

This fact also affects the character of the generation of X-ray radiation. Figure 3 shows the dependence of the average number of recorded quanta in one thermal cycle and the average value of the endpoint energy of the spectrum after each action of treatment on the crystal surface.

The results obtained make it possible to draw an unambiguous conclusion that mechanical processing of the free $Z$-surface reduces the intensity of $\mathrm{X}$-ray: the radiation yield drops almost 4 times after the first surface treatment with sandpaper and returns to the previous level only after treatment with polishing paste. However, an analysis of the endpoint energy of the spectrum shows that mechanical treatment raises the value of the endpoint energy from $45-50 \mathrm{keV}$ to $70-75 \mathrm{keV}$. The only exception is the point after processing with sandpaper with an average grain size of $6 \mu \mathrm{m}$, the energy rises to $60 \mathrm{keV}$. Polishing the surface lowers the endpoint energy to its original level. According to the results, surface treatment with an average grain size of 3 and $9 \mu \mathrm{m}$ makes it possible to obtain the endpoint energy larger, and an intermediate sanding between them at a grain size of $6 \mu \mathrm{m}$ made it possible to slightly increase the radiation yield withsome certain decreasing in the endpoint energy with respect to neighbouring points. While it is impossible to say unambiguously whether this effect is a consequence of the size of the processing grain, or the affect of the previous sanding on the subsequent.

Thus, the mechanical treatment of the z-surface substantially changes the spectrum of the generated Xray radiation in a pyroelectric source. Generation of radiation becomes less intense, but the spectrum becomes more high-energy. Apparently, roughness and unevenness on the surface of the crystal actually allows to obtain a locally stronger electric field, but this relief prevents the enhanced generation of particles.

Let us consider in more detail the X-ray spectra obtained at different states of the crystal surface (Fig. 4). During heating, the lines of niobium contained in the pyroelectric crystal ( $L_{\alpha}$-line $2.66 \mathrm{keV}, \quad K_{\alpha}$-line $16.62 \mathrm{keV}, K_{\beta}$-line $18.55 \mathrm{keV}$ ) were recorded. The heating corresponds to the positive charge polarity on the free surface of the crystal, the cooling corresponds to the negative polarity of the charge. By the endpoint energy of the spectrum, one can judge the maximum energy of electrons accelerated in a pyroelectric source. Heating corresponds to a positive charge polarity on the free surface of the crystal, cooling corresponds to a negative charge polarity. Figures 4 and 5 show that the 
increase in the endpoint energy of the spectrum occurs only with negative charge polarity, in the opposite case, both energy and intensity fall sharply. Consequently, the relief of the $Z$-surface affects the X-ray spectrum in a pyroelectric source differently depending on the polarity of the charge on the surface, and as a consequence, depending on the process leading to the generation of radiation. Apparently, the presence of a number of local irregularities on the surface of the crystal leads to an increase in the surface area of the crystal over which the charge is distributed, and as a consequence, a highly uneven charge density of the crystal, which is the reason for the weakening of the electric field near the crystal, weaker ionization of the mole- cules of the residual gas, and respectively, a generation $\mathrm{X}$-ray radiation. On the other hand, it is possible to obtain a higher electric potential on the formed local points, protrusions, which can contribute to a stronger electron emission from the crystal surface and their acceleration to higher energies than in the case of a flatter crystal surface. This may be the reason for the increase in the boundary energy of the spectrum in the case of cooling (corresponds to the negative polarity of the charge on the surface of the crystal)

Treatment with polishing paste makes the surface flatter, in this case, as in the case of the initial state of the surface, the radiation yield is higher, but the endpoint energy (and hence the electric potential) is less.
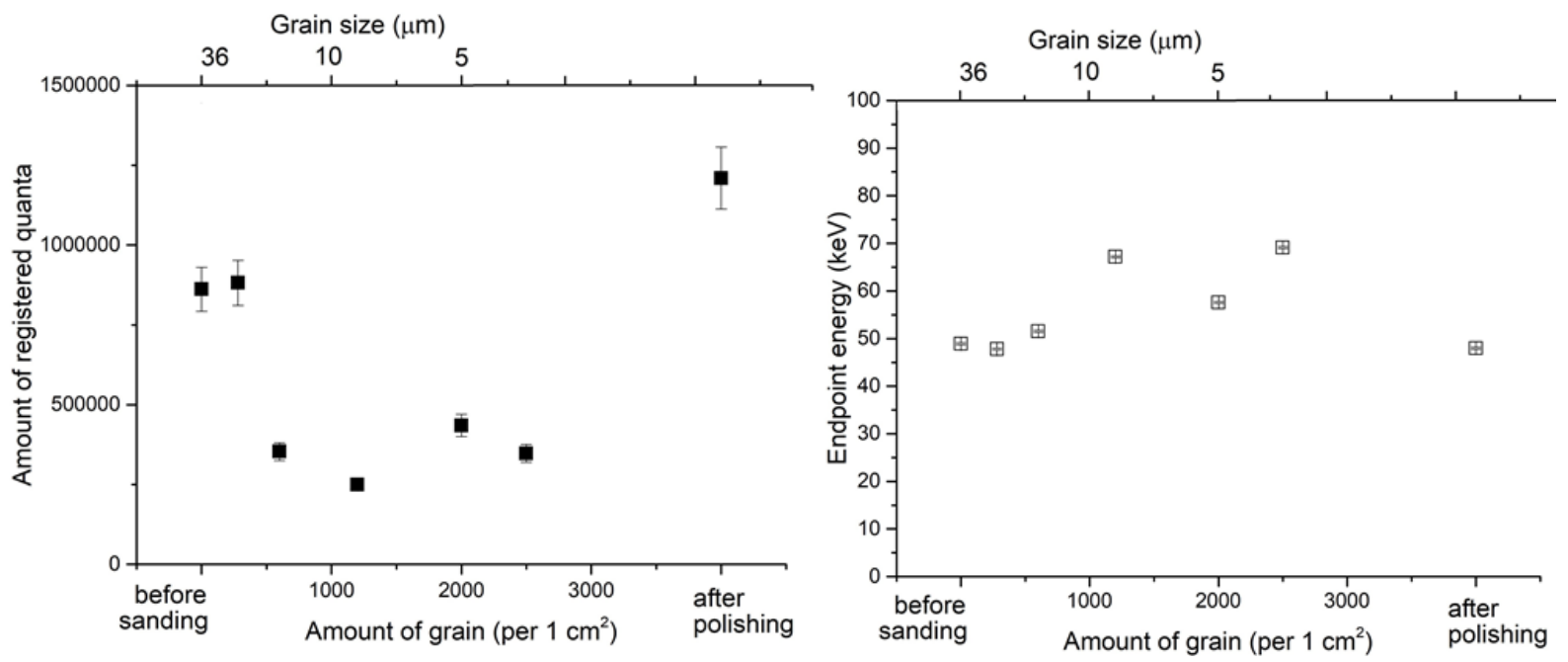

Fig. 3 - The dependence of the average number of recorded quanta in one thermal cycle (left) and the mean value of the endppoint energy of the spectrum (right) on the number of grains on the emery paper, and also before sanding and after polishing. The measurements were carried out consecutively from left to right
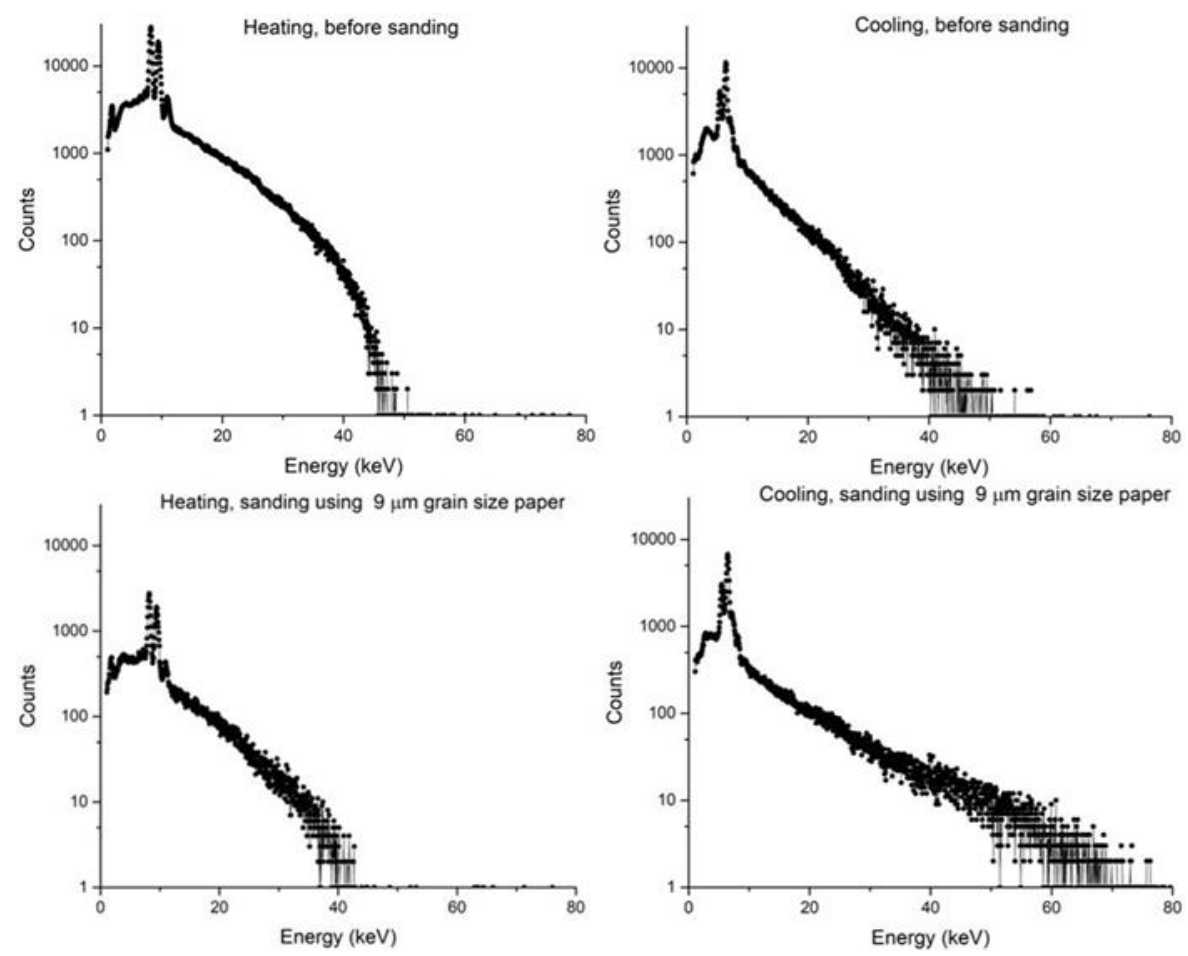

Fig. 4 - X-ray spectra under heating (left column) and cooling (right column) before machining the $Z$-surface of the crystal (top row), after sandpaper treatment with an average grain size of $9 \mu \mathrm{m}$ (bottom row) 

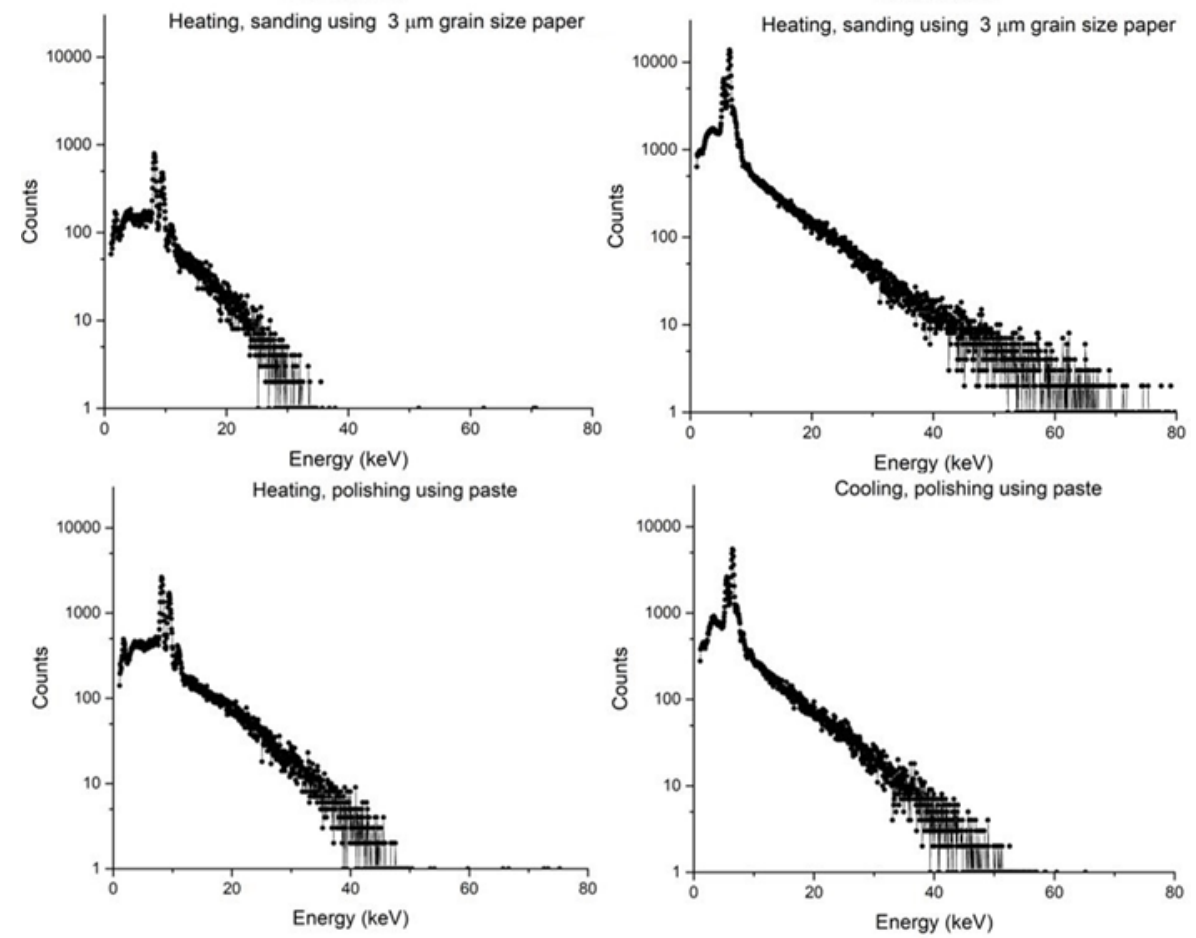

Fig. 5 - X-ray spectra under heating (left column) and cooling (right column) after sandpaper treatment with an average size grain $3 \mu \mathrm{m}$ (top row), after treatment with polishing paste (bottom row)

\section{CONCLUSION}

The results of the study show an unambiguous dependence of the generation of X-rays in the pyroelectric source on the relief of the free $Z$-surface of the pyroelectric crystal. The results of the study show an unambiguous dependence of the generation of X-rays in the pyroelectric source on the relief of the free $Z$-surface of the pyroelectric crystal. It should also be noted that there is no single-valued linear dependence on the intensity and the endpoint energy of radiation with a decrease in the grain size of the sandpaper. Polishing the surface leads to the removal of roughness on the surface of the crystal and to an increase in the yield and the endpoint energy of the X-ray radiation to the original level before treatment.

\section{REFERENCES}

1. J.D. Brownridge, S. Raboy, J. App. Phys. 86, 640 (1999),
2. V.I Nagaychenko, V.M. Sanin, A.M. Yegorov A.V. Shchagin, Probl. At. Sci. Technol. 43(2), 214 (2004).

3. www.amptek.com/coolx.html

4. V.I. Nagaichenko, V.A. Voronko, V.V. Sotnikov, V.V. Sidorenko, P.S. Kizim, A.M. Yegorov, A.V. Shchagin, Probl. At. Sci. Technol. 50(5), 72 (2008).
In general, the results obtained are consistent with the results of an earlier work [2], but a very important addition is the clearly demonstrated possibility of increasing the radiation energy for a negative charge polarity. This effect can be useful in the development of pyroelectric electron accelerators [6, 7]. However, mechanical treatment of the $Z$-surface does not give a global gain in the X-ray yield and cannot be considered as a way to improve pyroelectric sources.

\section{ACKNOWLEDGEMENTS}

The work was financially supported by a Program of the Ministry of Education and Science of the Russian Federation for higher education establishments, (project No.3.1631.2017/ПЧ)

5. V.I. Nagaychenko, V.V. Sotnikov, B.I. Ivanov, A.M. Yegorov, A.V. Shchagin, J. Surf. Invest. X-ray, Synchrotron Neutron Tech. 3, 81 (2007).

6. J.A. Geuther, Y. Danon, J. Appl. Phys. 97, 074109 (2005).

7. N. Kukhtarev, T. Kukhtareva, M. Bayssie, J. Wang, J.D. Brownridge, J. App. Phys. 96, 6794 (2004). 